\title{
A two-phase comprehensive NSCLC prognostic study identifies IncRNAs with significant main effect and interaction
}

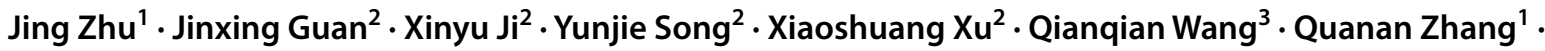 \\ Renhua Guo ${ }^{3} \cdot$ Rui Wang ${ }^{4} \cdot$ Ruyang Zhang ${ }^{2,4}$ (1)
}

Received: 18 August 2021 / Accepted: 2 February 2022 / Published online: 26 February 2022

(c) The Author(s) 2022

\begin{abstract}
Long noncoding RNA (lncRNA) are involved in regulating physiological behaviors for various malignant tumors, including non-small-cell lung cancer (NSCLC). However, few studies comprehensively evaluated both lncRNA-lncRNA interaction effects and main effects of lncRNA on overall survival of NSCLC. Hence, we performed a two-phase designed study of lncRNA expression in tumor tissues using 604 NSCLC patients from The Cancer Genome Atlas as the discovery phase and 839 patients from Gene Expression Omnibus as the validation phase. In the discovery phase, we adopted a two-step strategy, Screening before Testing, for dimension reduction and signal detection. These candidate lncRNAs first screened out by the weighted random forest (Ranger), were then tested through the Cox proportional hazards model adjusted for covariates. Significant lncRNAs with either type of effects aforementioned were carried forward into the validation phase to confirm their significances again. As a result, in the discovery phase, 19 lncRNAs were identified by Ranger, among which five lncRNAs and one pair of lncRNA-lncRNA interaction exhibited significant effects (FDR- $q \leq 0.05$ ) main and interaction effects on NSCLC survival, respectively, through Cox model. After the independent validation, we finally observed that one lncRNA (ENSG00000227403.1) with main effect was robustly associated with NSCLC prognosis $\left(H R_{\text {discovery }}=0.90, P=1.20 \times 10^{-3}\right.$; $H R_{\text {validation }}=0.94, P=4.11 \times 10^{-3}$ ) and one pair of IncRNAs (ENSG00000267121.4 and ENSG00000272369.1) had significant interaction effect on NSCLC survival $\left(H R_{\text {discovery }}=1.12, P=3.07 \times 10^{-4} ; H R_{\text {validation }}=1.11, P=0.0397\right)$. Our comprehensive NSCLC prognostic study of IncRNA provided population-level evidence for further functional study.
\end{abstract}

Keywords lncRNA $\cdot$ Main effect $\cdot$ Interaction $\cdot$ Non-small-cell lung cancer $\cdot$ Overall survival

Communicated by Shuhua Xu.

Jing Zhu, Jinxing Guan and Xinyu Ji contributed equally to this work.

Quanan Zhang

zhang_quan_an_njmu@163.com

Renhua Guo

rhguo@njmu.edu.cn

Rui Wang

wangrui218@163.com

Ruyang Zhang

zhangruyang@njmu.edu.cn

1 Department of Oncology, The Affiliated Jiangning Hospital of Nanjing Medical University, 169 Hushan Road, No. 2 Building, 212 East Ward, Nanjing 211100, Jiangsu, China

2 Department of Biostatistics, Center for Global Health, School of Public Health, Nanjing Medical University, 101 Longmian
Avenue, SPH Building, Room 406, Nanjing 211166, Jiangsu, China

3 Department of Medical Oncology, The First Affiliated Hospital of Nanjing Medical University, 300 Guangzhou Road, No. 3 Building, Floor 10, Nanjing 210003, Jiangsu, China

4 Department of Medical Oncology, Jinling Hospital, School of Medicine, Nanjing University, 34 Yanggongjing Street, Building 1, Floor 6, Nanjing 210002, Jiangsu, China 


\section{Introduction}

Lung cancer is the leading cause of cancer death worldwide (Sung et al. 2021). It is well known that majority of lung cancers are non-small cell lung cancer (NSCLC) in histological classification, including lung adenocarcinoma (LUAD) and lung squamous cell carcinoma (LUSC) (Travis 2020). In the half past century, great efforts have been made in the treatments of lung cancer, including surgery, chemotherapy, radiotherapy, targeted therapy, anti-angiogenic therapy, immunotherapy, etc. (Mok et al. 2009, 2019; Auperin et al. 2010). Therefore, the survival rate and quality of life of patients has been significantly improved (Wu et al. 2014; Mok et al. 2019; Sung et al. 2021). However, pathological mechanism of lung cancer progression still largely remains unclear (e.g. tumor cell invasion and drug resistance) (Herbst et al. 2018).

Long noncoding RNAs (lncRNAs) are a type of nonprotein-coding transcript longer than 200 nucleotides and are one of the emerging regulators which are involved in diverse biological processes (Boon et al. 2016; Jandura and Krause 2017). Many studies have demonstrated that lncRNA plays an important role (e.g. oncogenes or tumor suppressors) in regulating the physiological behaviors of malignant tumors, including lung cancer (Schmitt and Chang 2016; Braicu et al. 2019; Xu et al. 2019). In recent years, accumulating evidences indicate that the interaction effects of lncRNAs are essential for the initiation and progression of cancers, such as lncRNA-protein interaction and lncRNAmiRNA-mRNA network (Ferrè et al. 2016; Wang et al. 2019). Especially, lncRNA-AC020978/PKM2/HIF-1 $\alpha$ is a new perspective in the prevention or treatment of NSCLC (Hua et al. 2020). However, few studies ever focused on lncRNA-lncRNA interaction effects on NSCLC overall survival, which may provide pivotal clues for the biologic mechanisms of complex diseases (Zhang et al. 2019b) and enhance prediction accuracy (Chatterjee et al. 2016; Li et al. 2019).

Hence, we performed a comprehensive analysis of lncRNAs to evaluate their main effects and interaction effects on NSCLC survival through a two-phase designed study, using 604 NSCLC patients from The Cancer Genome Atlas (TCGA) as the discovery phase and 839 patients from Gene Expression Omnibus (GEO) as the validation phase.

\section{Materials and methods}

\section{Data collection and study population}

LncRNA expression data in tumor tissue, as well as demographic and clinical data, were retrieved from TCGA and GEO. (1) TCGA: The fragments per kilobase of per million
(FPKM) value of lncRNA expression, as well as corresponding demographic and clinical information of 986 NSCLC patients, were downloaded from TCGA (https://portal.gdc. cancer.gov). (2) GEO: Totally, 6 available datasets consisting of 1096 NSCLC patients, profiled by the Affymetrix Human Genome U133A Plus 2.0 Array, were obtained from GEO (https://www.ncbi.nlm.nih.gov/geo/), which had the maximum overlapped IncRNAs to TCGA data, adequate sample sizes after quality control (e.g., $N>50$ ) and, meanwhile raw data instead of standardized data, including GSE3141, GSE37745, GSE30219, GSE50081, GSE29013 and GSE31210.

LncRNA was annotated by gencode.v22 (https://www. gencodegenes.org/), and we obtained expression of 15,900 IncRNAs in TCGA. Before association analysis, we performed quality control procedures to acquire reliable lncRNA expression. Briefly, lncRNAs were excluded if they met any of the below criteria: all gene expression values equal to 0 or proportion of missing values is greater than $10 \%$. Further, samples with missing values of any clinical variables were also excluded. Finally, 604 samples (294 LUAD and 310 LUSC) with 4313 lncRNAs in TCGA were retained in subsequent association analysis. In GEO, there were 839 patients (634 LUAD and 205 LUSC) remained after removing patients without complete clinical information. We performed a two-phase designed study of IncRNAs using subjects in TCGA as the discovery phase and subjects in GEO as the validation phase. The demographic and clinical information of subjects from two phases were described in Table 1.

\section{Statistical analysis}

The entire statistical analysis workflow was given in Fig. 1 .

\section{Screening before Testing strategy in the discovery phase}

In the discovery phase, we adopted a two-step strategy, named Screening before Testing, for dimension reduction and signal detection. In the screening step, Ranger, a weighted version of random forest for analyzing time-toevent data, while adjusted for covariates, was employed to evaluate the importance of each individual lncRNA (Breiman 2001), using R package ranger. A weight of $100 \%$ was assigned to each covariate to ensure all covariates were adjusted in each classification tree, including age, gender, race, clinical stage, smoking status and pack-year of smoking. Variable importance score (VIS) of each lncRNA was estimated and ranked in a descending order. The sliding windows sequential forward feature selection (SWSFS) algorithm was then applied to identify the top important lncRNAs (Jiang et al. 2009). The SWSFS algorithm incorporated 
Table 1 Demographic and clinical descriptions of NSCLC patients in the discovery and validation phases

\begin{tabular}{|c|c|c|}
\hline Variable & Discovery phase $(N=604)$ & Validation phase $(N=839)$ \\
\hline Age (years) & $66.02 \pm 9.55$ & $63.51 \pm 9.34$ \\
\hline \multicolumn{3}{|l|}{ Gender, $N(\%)$} \\
\hline Male & $355(58.77 \%)$ & $411(56.38 \%)$ \\
\hline Female & $249(41.23 \%)$ & $318(43.62 \%)$ \\
\hline Unknown & 0 & 110 \\
\hline \multicolumn{3}{|l|}{ Race, $N(\%)$} \\
\hline Asian & $8(1.32 \%)$ & 0 \\
\hline Black & $55(9.11 \%)$ & 0 \\
\hline White & $541(89.57 \%)$ & 0 \\
\hline Unknown & 0 & 839 \\
\hline \multicolumn{3}{|l|}{ Smoke status, $N(\%)$} \\
\hline Never & 0 & $26(6 \%)$ \\
\hline Former & $416(68.87 \%)$ & $243(56.12 \%)$ \\
\hline Current & $188(31.13 \%)$ & $164(37.88 \%)$ \\
\hline Unknown & 0 & 406 \\
\hline Smoking packs every year & $47.38 \pm 29.96$ & - \\
\hline \multicolumn{3}{|l|}{ Clinical stage, $N(\%)$} \\
\hline I & $328(54.30 \%)$ & $496(68.04 \%)$ \\
\hline II & $171(28.31 \%)$ & $172(23.59 \%)$ \\
\hline III & $88(14.57 \%)$ & $53(7.27 \%)$ \\
\hline IV & $17(2.81 \%)$ & $8(1.10 \%)$ \\
\hline Unknown & 0 & 110 \\
\hline \multicolumn{3}{|l|}{ Histology, $N(\%)$} \\
\hline LUAD & $294(48.68 \%)$ & $634(75.57 \%)$ \\
\hline LUSC & $310(51.32 \%)$ & $205(24.43 \%)$ \\
\hline \multicolumn{3}{|l|}{ Survival year } \\
\hline Median (95\% CI) & $3.91(3.33-4.93)$ & $7.53(6.30-8.92)$ \\
\hline Censoring rate & $59.60 \%$ & $55.66 \%$ \\
\hline
\end{tabular}

The validation phase consists of six datasets from GEO, including GSE3141 ( $N=110)$, GSE37745 $(N=172), \operatorname{GSE} 30219(N=104), \operatorname{GSE} 50081(N=172), \operatorname{GSE} 31210(N=226)$ and GSE29013 $(N=55)$ these IncRNAs one by one into the Ranger model by the order of VIS. Then, we plotted the out of bagging (OOB) error rate, which measured the performance of each model consisting of top $k \operatorname{lncRNAs}$. The top candidate lncRNAs were screened out for further analysis when the Ranger model reaching the lowest $\mathrm{OOB}$ error rate.

In the testing step, we further evaluated both main effects and lncRNA-lncRNA interaction effects of these top candidate IncRNAs, through Cox proportional hazards model adjusted for the same covariates aforementioned, using the $\mathrm{R}$ package survival. For main effect analysis and interaction effect analysis, we adopted the model 1 and 2 below, respectively.
The association results were described as hazard ratio (HR) and 95\% confidence interval (CI). Multiple comparisons were adjusted using false discovery rate method (FDR; measured by FDR- $q$ value) (Klipper-Aurbach et al. 1995). LncRNAs with significant (FDR- $q \leq 0.05$ ) main effects or interaction effects on NSCLC survival were reserved for subsequent analysis.

To retain the robustly significant association between lncRNA and NSCLC survival, we additionally downloaded raw counts of IncRNA expression from TCGA and calculated a trimmed mean of $M$-values (TMM) between each pair of samples to adjust the library sizes (Robinson and Oshlack 2010), by R package DGEobj.utils. Then,

$h(t)=h_{0}(t) \exp \left(\alpha_{1} \times \operatorname{lncRNA}+\sum \beta_{i} \times\right.$ Covariate $\left._{i}\right)$
$h(t)=h_{0}(t) \exp \left(\alpha_{1} \times \operatorname{lncRNA}_{1}+\alpha_{2} \times \operatorname{lncRNA}_{2}+\alpha_{3} \times \operatorname{lncRNA}_{1} \times \operatorname{lncRNA}_{2}+\sum \beta_{i} \times\right.$ Covariate $\left._{i}\right)$ 


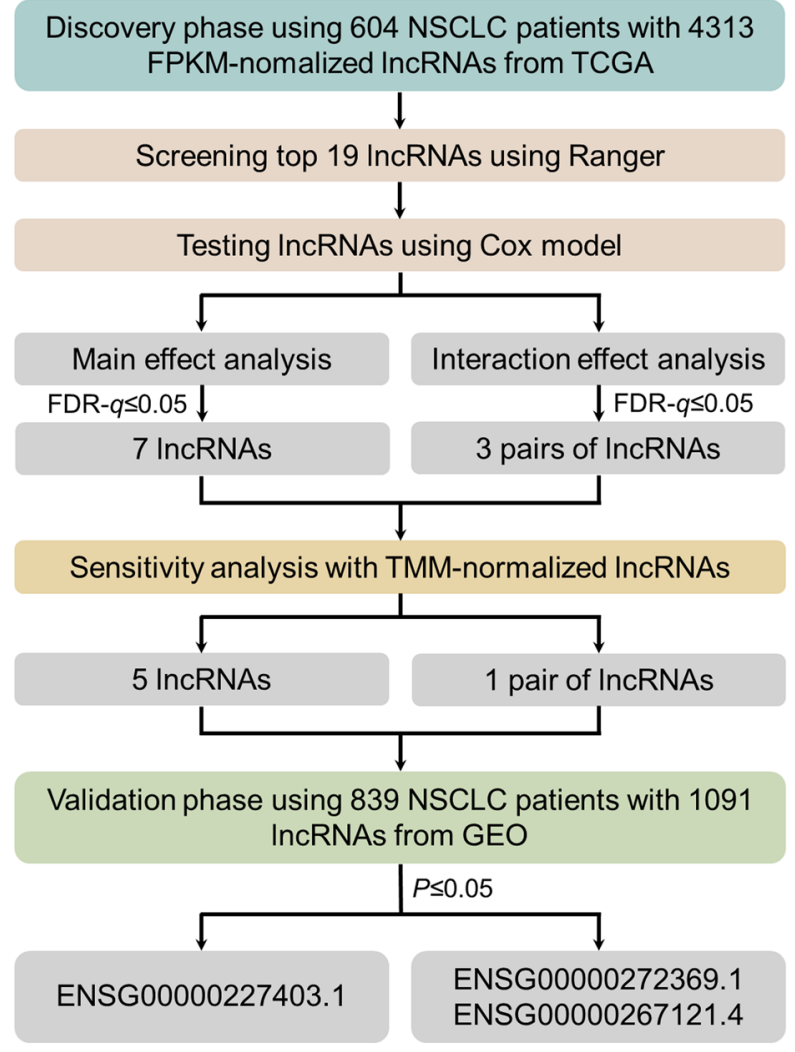

Fig. 1 Statistical analysis work flow

we performed sensitivity analysis of TMM-normalized expression data for top candidate IncRNAs and significant (FDR- $q \leq 0.05$ ) lncRNAs with either type of effects aforementioned were preserved. Finally, only these lncRNAs simultaneously significant in both FPKM and TMM data were carried forward into the validation phase to confirm their significances once again.

\section{Trans-platform pseudo-validation strategy in the validation phase}

Due to the difference of gene expression platforms between TCGA and GEO, some significant lncRNAs identified in TCGA were not profiled in GEO. Therefore, we performed trans-platform pseudo-validation using their surrogate lncRNAs, which had significant (FDR- $q \leq 0.05$ ) and maximum correlation with the targets. The main effects and interaction effects of these surrogate lncRNAs were again tested by Cox proportional hazards model adjusted for covariates. Finally, only these IncRNAs were preserved if they met both criteria below: (1) $P \leq 0.05$ in the validation phase, and (2) consistent effects across both discovery and validation phases.

Furthermore, the significances of these lncRNAs were evaluated in LUAD and LUSC subgroup populations.
Besides, we compared two models to highlight the contribution of lncRNAs to the prognostic prediction of NSCLC survival: (1) a basic model with merely demographic and clinical information, and (2) an optimized model added significant lncRNAs with either main effects or interaction effects. We predicted 3- and 5-year overall survival of NSCLC patients using the Kaplan-Meier method for timeto-event data (Heagerty et al. 2000). The accuracy of the prediction was presented using a receiver operating characteristic (ROC) curve and was measured by time-dependent area under the ROC curve (AUC) by the R package survivalROC. The $95 \% \mathrm{CI}$ and $P$ value of the AUC improvement were calculated on the basis of 1,000-time bootstrap resampling.

Continuous variables were summarized as mean \pm standard deviation (SD), and categorized variables were described by frequency $(n)$ and proportion (\%). Statistical analyses were performed using $\mathrm{R}$ version 3.6.3 (The R Foundation of Statistical Computing, Vienna, Austria).

\section{Result}

\section{The two-phase study identified one IncRNA with significant main effect and one pair of IncRNAs with significant interactions}

In the discovery phase, we identified top 19 lncRNAs which together had the lowest OOB error in the screening step by the SWSFS algorithm (Figure S1, S2). In the testing step, we observed five IncRNAs (ENSG00000227403.1, ENSG00000273038.2， ENSG00000269609.4, ENSG00000273230.1 and ENSG00000204949.7) and one pair of lncRNAs (ENSG00000272369.1 and ENSG00000267121.4) had robust and significant (FDR$q \leq 0.05$ ) main effects and interaction effects on NSCLC survival, respectively (Table S1). The annotation information was presented in Table S2. Compared to the basic model, the optimized model added these lncRNAs with either type of effects had significant improved prediction accuracy (3-year survival: $\mathrm{AUC}_{\text {optimized }}=0.72 \mathrm{vs} \mathrm{AUC}_{\mathrm{basic}}=0.65,12.0 \%$ increase, $P<2.2 \times 10^{-16} ; 5$-year survival: $\mathrm{AUC}_{\text {optimized }}=0.74$ vs $\mathrm{AUC}_{\text {basic }}=0.63,16.9 \%$ increase, $P<2.2 \times 10^{-16}$ ) (Figure S3).

In the validation phase (Table S3), we finally observed one lncRNA, ENSG00000227403.1, significantly associated with NSCLC survival $\left(H R_{\text {discovery }}=0.90, P=1.20 \times 10^{-3}\right.$; $\left.H R_{\text {validation }}=0.94, P=4.11 \times 10^{-3}\right)$ and one pair of $1 \mathrm{ncR}$ NAs (ENSG00000267121.4 and ENSG00000272369.1) had significant interaction effect on NSCLC survival $\left(H R_{\text {discovery }}=1.12, \mathrm{P}=3.07 \times 10^{-4} ; H R_{\text {validation }}=1.11\right.$, $P=0.0397$ ) (Table 2). 
Table 2 Association results of one lncRNA and one pair of IncRNAs derived from Cox proportional hazards model adjusted for covariates in main effect and interaction effect analyses

\begin{tabular}{lllllll}
\hline Type of analysis & LncRNA & \multicolumn{2}{l}{ Discovery phase } & & \multicolumn{2}{l}{ Validation phase } \\
\cline { 6 - 7 } \cline { 6 - 7 } & & HR $(95 \%$ CI $)$ & $P$ & & HR $(95 \%$ CI $)$ & $P$ \\
\hline Main effect & ENSG00000227403.1 & $0.90(0.84,0.96)$ & $1.20 \times 10^{-3}$ & & $0.94(0.90,0.98)$ & $4.11 \times 10^{-3}$ \\
Interaction effect & ENSG00000272369.1 & $0.69(0.57,0.83)$ & $8.28 \times 10^{-5}$ & & $0.44(0.24,0.79)$ & $6.41 \times 10^{-3}$ \\
& ENSG00000267121.4 & $1.30(1.12,1.51)$ & $5.61 \times 10^{-4}$ & & $0.38(0.17,0.88)$ & 0.0228 \\
& Interaction term & $1.12(1.05,1.19)$ & $3.07 \times 10^{-4}$ & & $1.11(1.01,1.23)$ & 0.0397 \\
\hline
\end{tabular}

In the validation phase, the surrogates of ENSG00000227403.1, ENSG00000272369.1 and ENSG00000267121.4 were ENSG00000253738.1, ENSG00000265666.1 and ENSG00000227039.5, respectively
Fig. 2 Kaplan-Meier survival curves for patients with low and high expression of ENSG00000227403.1 in the discovery and validation phases
(A) Discovery phase

ENSG00000227403.1

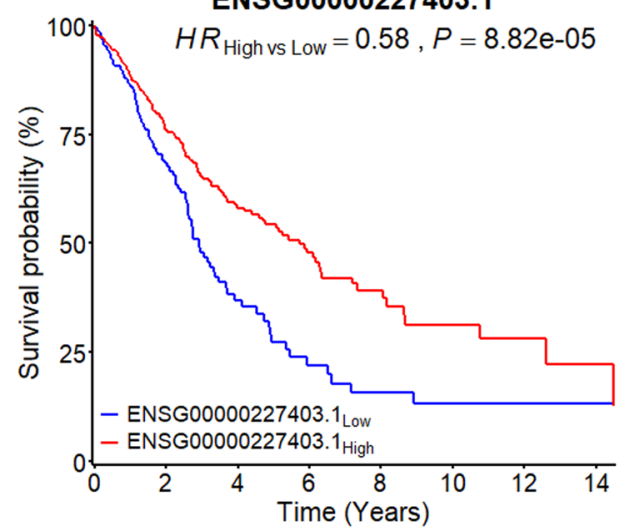

(B) Validation phase

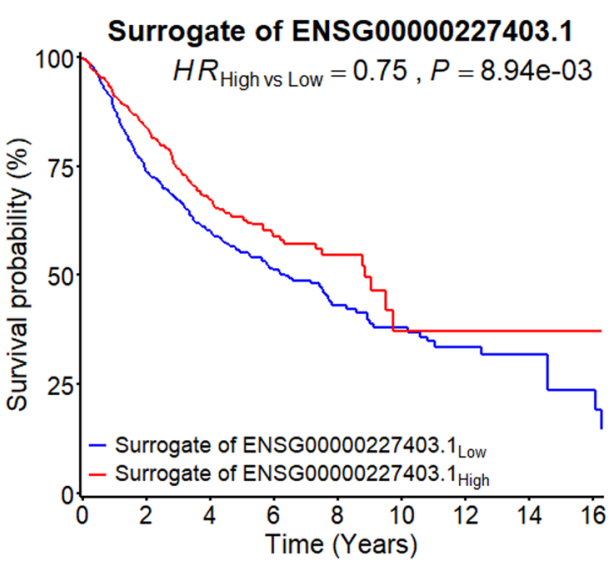

\section{The IncRNA with significant main effect substantially discriminated subjects at high risk of mortality from NSCLC patients}

NSCLC patients were divided into low and high expression groups based on median value of ENSG00000227403.1. By comparison of the Kaplan-Meier survival curves between two groups (Fig. 2), these patients in high expression group had significant better overall survival in the discovery phase $\left(H R_{\text {high vs low }}=0.58, P=8.82 \times 10^{-5}\right)$ and the validation phase $\left(H R_{\text {high vs low }}=0.75, P=8.94 \times 10^{-3}\right)$, indicating that subjects with low expression of ENSG00000227403.1 were at high risk of mortality.

\section{The effect of one IncRNA on NSCLC survival was modified by another IncRNA}

For the interaction between ENSG00000272369.1 and ENSG00000267121.4, we observed that, with increased expression level of ENSG00000267121.4, there was an elevated effect of ENSG00000272369.1 on NSCLC survival in the discovery phase (Fig. 3A) and the validation phase (Fig. 3B). Therefore, ENSG00000267121.4 was a modifier of the association between ENSG00000272369.1 and NSCLC survival. Besides, to better understand this interaction, patients were divided into low and high expression groups based on their ENSG00000267121.4 values, using cutoff value 3.26 and 7.88 in the discovery and validation phase, respectively. We observed varied effects of ENSG00000272369.1 between different ENSG00000267121.4 expression groups. High expression of ENSG00000272369.1 exhibited a significantly protective effect on NSCLC for these patients in low expression group of ENSG00000267121.4 in the discovery phase $(H R=0.86, P=0.0309)$ and the validation phase $(H R=0.73$, $\left.P=3.33 \times 10^{-5}\right)($ Fig. $3 \mathrm{C}, \mathrm{D})$. However, the effect of ENSG00000272369.1 was reversed for these patients in high expression group of ENSG00000267121.4 in the discovery phase $(H R=1.31, P=0.0171)$ and the validation phase $(H R=1.17, P=0.7123)$.

To visualize the heterogeneous effect of ENSG00000272369.1 between two expression groups of ENSG00000267121.4, we further categorized ENSG00000272369.1 into dichotomous variable by its median value. In low expression group of ENSG00000267121.4, subjects with high expression of ENSG00000272369.1 had significantly better survival compared to these with low ENSG00000272369.1 in the discovery phase $\left(H R_{\text {high vs low }}=0.72, P=0.0458\right)($ Fig. $4 \mathrm{~A})$ 


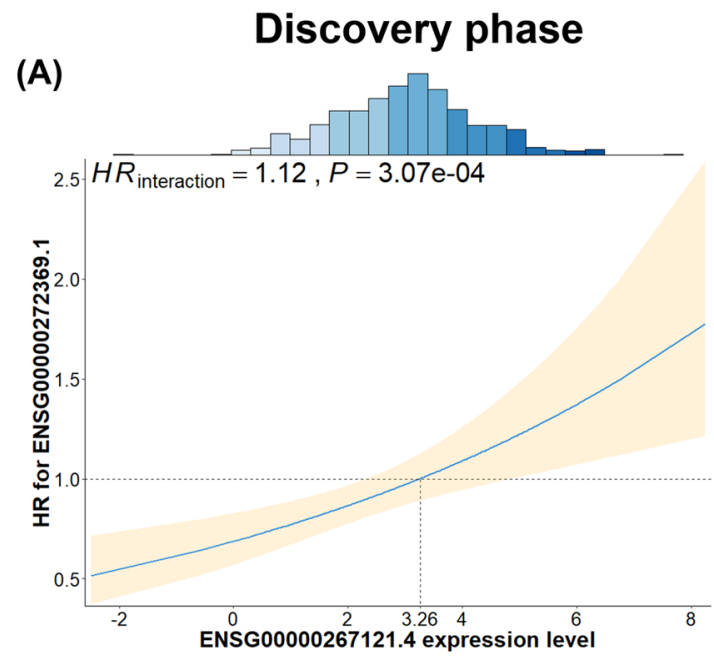

(C)

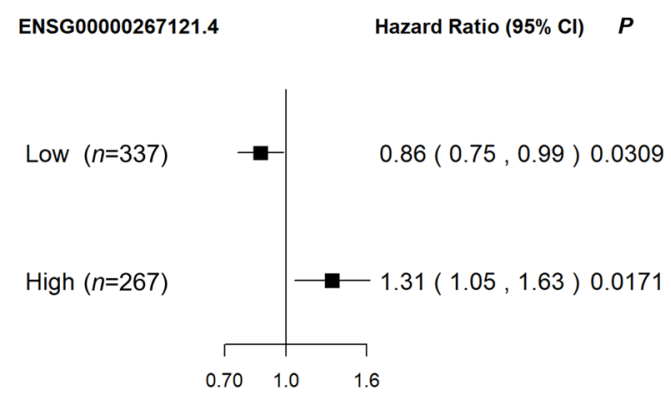

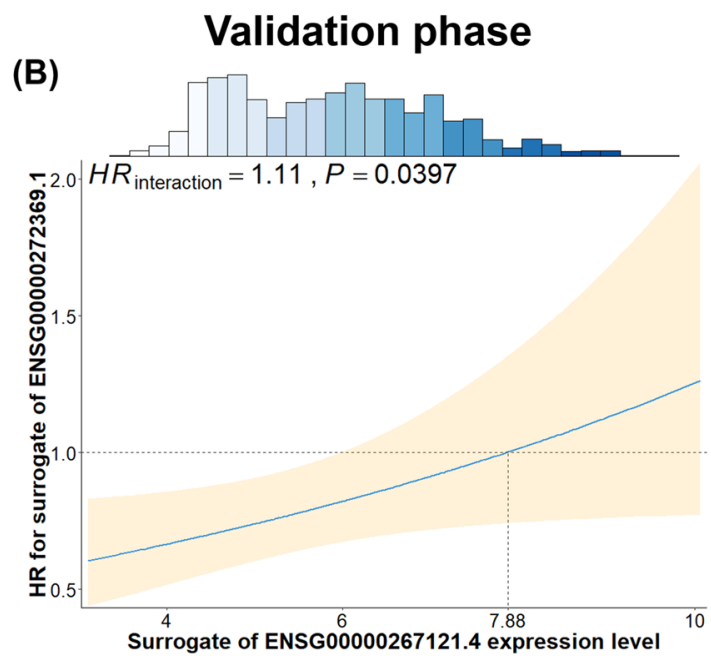

(D)

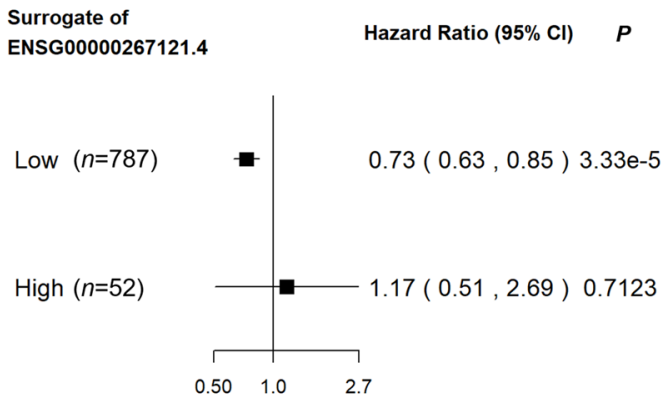

Fig. 3 Line and forest plots illustrating lncRNA-lncRNA interaction effect between ENSG00000267121.4 and ENSG0000027236.9 on NSCLC survival

and the validation phase $\left(H R_{\text {high vs low }}=0.66, P=1.57 \times 10^{-4}\right)$ (Fig. 4C). On the contrary, in high expression group of ENSG00000267121.4, subjects with high expression of ENSG00000272369.1 had worse survival in two phases (Fig. 4B, D).

The stratified analysis by histology confirmed the significance of main effect of ENSG00000227403.1 on NSCLC survival (Table S4), except for LUSC subgroup with small sample size in the validation phase. Besides, the interaction effect between ENSG00000272369.1 and ENSG00000267121.4 maintained significance only in LUAD subgroup in the discovery phase.

\section{Discussion}

Non-coding RNAs (ncRNAs), accounting for $98 \%$ of the human genome, are classified into several types including: lncRNAs, micro RNAs (miRNAs), circular RNA (circRNAs) and so on (Zhang et al. 2020a; b). Human lncRNAs are abundant and diverse, and nearly 53,000 different lncRNAs are known but only about 1000 are present in sufficiently high copy number to authentically justify their functional importance (Djebali et al. 2012). In recent years, extensive evidence has suggested that lncRNAs are involved in the occurrence of many diseases, including cancer (Liang et al. 2018). LncRNAs have been shown to participate in the development, progression, proliferation, and invasion of NSCLC used variety of ways (Zhang et al. 2019c; Chen et al. 2020a, b).

Emerging evidence indicates that there exist many types of interactions associated with NSCLC survival, including gene-gene (Zhang et al. 2019b), gene-smoking (Zhang et al. 2019a), gene-age (Chen et al. 2020a; b) and gene-histology interactions ( $\mathrm{Ji}$ et al. 2020). As is well known, interactions provide important clues for the biologic mechanisms and heritability of complex diseases (Trerotola et al. 2015). Thus, biomarkers with main effects only explained a small proportion of the phenotypic variations and the GxG interactions may be one of the important reasons accounting for the missing heritability (Trerotola et al. 2015). The studies of lncRNAs account for $13 \%$ of the total studies of ncRNAs in lung cancer until year 2019 (Braicu et al. 2019). Anyway, 
Fig. 4 Kaplan-Meier survival curves for patients having different expression levels of two lncRNAs

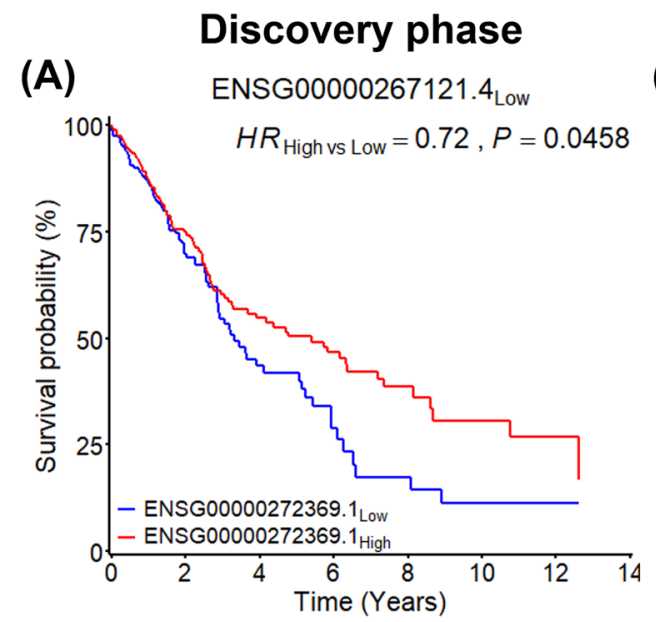

(B)
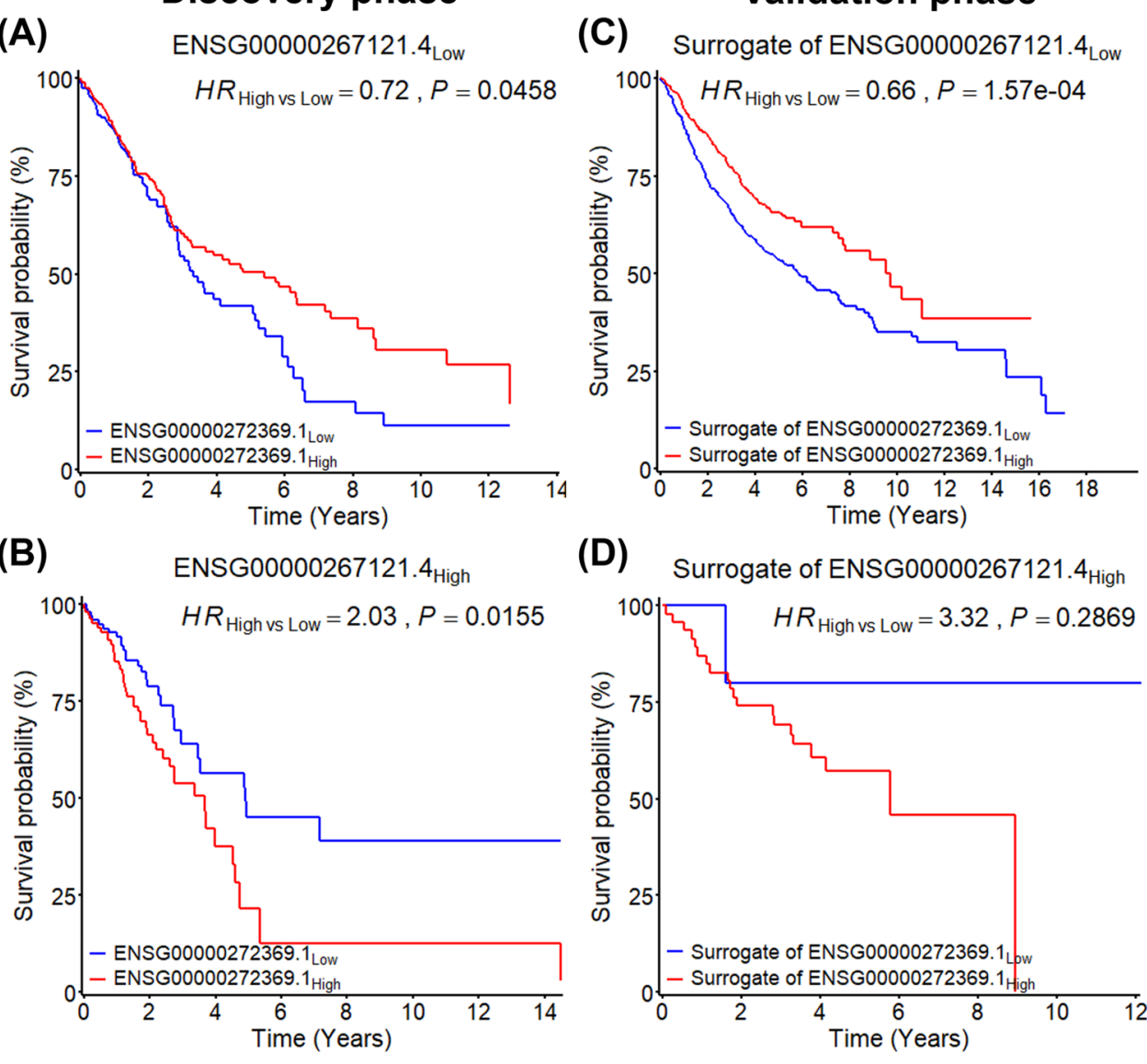

(D) Surrogate of ENSG00000267121.4 $4_{\text {High }}$

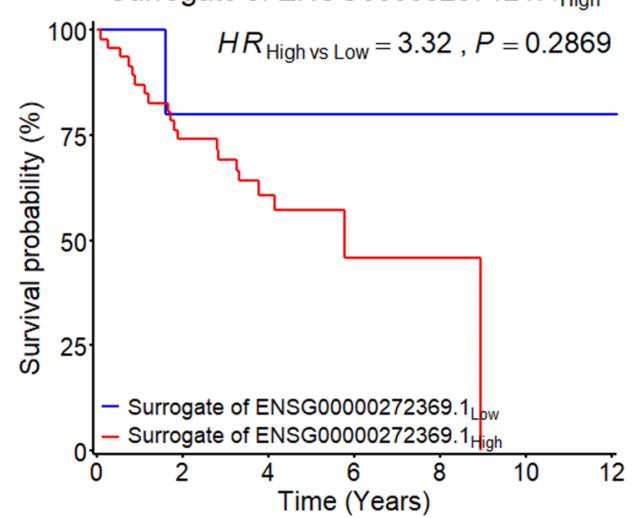

a mass of studies only focused on the association between IncRNAs and lung cancer, by testing their main effects. GxG interaction analysis provides a different way of identifying new biomarkers (Cordell 2009), which can further improve the predictive accuracy of statistical models (Cordell 2009; Zhang et al. 2020a, b), or offer statistical evidence for functional studies. To our knowledge, this perhaps was the first attempt to explore the association between lncRNA-IncRNA interaction and NSCLC survival in population level. But functional experiments are still warranted to elaborate underlying mechanism.

For the three IncRNAs (ENSG00000227403.1, ENSG00000267121.4 and ENSG00000272369.1) successfully validated in an independent population, previous study indicated that AC009299.3, to which ENSG00000227403.1 mapped, was involved in the control of autophagy (Zhu et al. 2018; Wu et al. 2021). Meanwhile, high expression of this gene was also associated with better LUAD prognosis (Wu et al. 2021). Besides, CTD-2020K17.1, where ENSG00000267121.4 located, has been identified to promote migration, invasion, and proliferation of ovarian cancer (Zhu et al. 2018). Although these three IncRNAs lack explicit functional level evidence relevant to NSCLC survival, we provided robust and significant population-level evidence for further mechanistic study.

For other four IncRNAs (ENSG00000204949.7, ENSG00000273038.2, ENSG00000269609.4 and ENSG00000273230.1) failed in the independent validation, FAM83A-AS1 (ENSG00000204949.7) and RP11$479 G 22.8$ (ENSG00000273038.2) play essential roles in the development and progression of several cancers, including lung cancer, esophageal cancer and hepatocellular carcinoma (Wei and Zhang 2016; Wu et al. 2018; $\mathrm{He}$ and Yu 2019; Shi et al. 2019; Huang et al. 2020; Jia et al. 2021). Besides, RPARP-ASI (ENSG00000269609.4) could promote the proliferation, migration and invasion of tumor cells through sponging mir-125a-5p, which had been proved functional in the growth, invasion and metastasis of lung cancer and other cancers (Naidu et al. 2017; Ren et al. 2021).

Our study has several strengths. First, our study simultaneously evaluated both main effects of lncRNAs and lncRNA-lncRNA interaction effects on NSCLC survival, which was a comprehensive prognostic study of lncRNAs. And, to our knowledge, this is perhaps the first lncRNA-lncRNA interaction study of NSCLC survival. Besides one lncRNA with significant main effect, we 
additionally identified one pair of lncRNAs which exhibited significant interaction effect, providing potential evidence that complex disease (e.g., lung cancer) was driven by complex association pattern. Second, we utilized a two-phase study design to control the false positives, where statistical significance was corrected using FDR method in the discovery phase and again confirmed in the validation phase. Third, we adopted a two-step strategy, Screening before Testing, to improve the calculation speed of analysis and boost the statistical power of analysis in high dimensional scenario. Meanwhile, covariates were adjusted in both screening and testing steps to obtain more roust association results.

We also acknowledge some limitations. First, the significant lncRNAs identified in TCGA happen to be not profiled in GEO. Thus, we compromised by a trans-platform pseudo-validation using surrogate lncRNAs of these target lncRNAs. Even though one lncRNA and one pair of lncRNAs were successfully validated, additional available public database and further studies of target lncRNAs are still warranted. Second, the censoring rate of time-to-event data is high in TCGA and GEO, which may result in low statistical power in analysis. Thus, we only focused on two-way interaction between pair of lncRNAs. Third, further functional experiments of IncRNAs are warranted to provide biological evidence, beyond our statistical evidence. Thus, the association still should be interpreted with caution.

\section{Conclusion}

Our two-phase comprehensive NSCLC prognostic study of lncRNAs identified one lncRNA (ENSG00000227403.1) with significant main effects and one pair of lncRNAs (ENSG00000267121.4 and ENSG00000272369.1) with significant interaction effects on overall survival, providing population-level evidence for further functional study.

Supplementary Information The online version contains supplementary material available at https://doi.org/10.1007/s00438-022-01869-3.

Acknowledgements The authors thank TCGA and GEO for contributing demographic, clinical, and gene expression data, as well as all subjects who participated in these study cohorts.

Author contributions RZ, RG, RW, QZ and JZ contributed to the study design. RZ, JZ, JG and JX contributed to data collection. JZ, JG, JX, RW and YS performed statistical analysis and interpretation. RZ, JZ, JG, JX, XX and QW drafted and revised the manuscript. All authors contributed to critical revision of the manuscript and approved its final version. Financial support and study supervision were provided by RZ and RG.
Funding This study was funded by the Natural Science Foundation of Jiangsu Province (BK20191354 to R. Z.), China Postdoctoral Science Foundation (2018M633767 to R. Z.), National Natural Science Foundation of China (81772995 to R. W. and 81972188 to R. G.), and Priority Academic Program Development of Jiangsu Higher Education Institutions (PAPD). R. Z. was partially supported by the Qing Lan Project of the Higher Education Institutions of Jiangsu Province and the Outstanding Young Teachers Training Program of Nanjing Medical University.

Availability of data and material The datasets used in the current study are available from the corresponding author on reasonable request.

Code availability The datasets used in the current study are available from the corresponding author on reasonable request.

\section{Declarations}

Conflicts of interest The authors declare that they have no conflict of interest.

Ethical approval NA.

Consent to participate NA.

Consent for publication All authors have reviewed the manuscript and consented for publication.

Open Access This article is licensed under a Creative Commons Attribution 4.0 International License, which permits use, sharing, adaptation, distribution and reproduction in any medium or format, as long as you give appropriate credit to the original author(s) and the source, provide a link to the Creative Commons licence, and indicate if changes were made. The images or other third party material in this article are included in the article's Creative Commons licence, unless indicated otherwise in a credit line to the material. If material is not included in the article's Creative Commons licence and your intended use is not permitted by statutory regulation or exceeds the permitted use, you will need to obtain permission directly from the copyright holder. To view a copy of this licence, visit http://creativecommons.org/licenses/by/4.0/.

\section{References}

Auperin A, Le Pechoux C et al (2010) Meta-analysis of concomitant versus sequential radiochemotherapy in locally advanced nonsmall-cell lung cancer. J Clin Oncol 28(13):2181-2190. https:// doi.org/10.1200/JCO.2009.26.2543

Boon RA, Jaé N et al (2016) Long noncoding RNAs: from clinical genetics to therapeutic targets? J Am Coll Cardiol 67(10):12141226. https://doi.org/10.1016/j.jacc.2015.12.051

Braicu C, Zimta AA et al (2019) The function of non-coding RNAs in lung cancer tumorigenesis. Cancers. https://doi.org/10.3390/ cancers 11050605

Breiman L (2001) Random forests. Mach Learn 45(1):5-32. https:// doi.org/10.1023/A:1010933404324

Chatterjee N, Shi J et al (2016) Developing and evaluating polygenic risk prediction models for stratified disease prevention. Nat Rev Genet 17(7):392-406. https://doi.org/10.1038/nrg.2016.27

Chen C, Wei Y et al (2020a) Epigenome-wide gene-age interaction analysis reveals reversed effects of PRODH DNA methylation on 
survival between young and elderly early-stage NSCLC patients. Aging 12(11):10642-10662. https://doi.org/10.18632/aging. 103284

Chen Z, Chen X et al (2020b) Integrative analysis of NSCLC identifies LINC01234 as an oncogenic lncRNA that interacts with HNRNPA2B1 and regulates miR-106b biogenesis. Mol Ther 28(6):1479-1493. https://doi.org/10.1016/j.ymthe.2020.03.010

Cordell HJ (2009) Detecting gene-gene interactions that underlie human diseases. Nat Rev Genet 10(6):392-404. https://doi.org/ $10.1038 / \operatorname{nrg} 2579$

Djebali S, Davis CA et al (2012) Landscape of transcription in human cells. Nature 489(7414):101-108. https://doi.org/10.1038/natur e11233

Ferrè F, Colantoni A et al (2016) Revealing protein-lncRNA interaction. Brief Bioinform 17(1):106-116. https://doi.org/10.1093/bib/ bbv031

He J, Yu J (2019) Long noncoding RNA FAM83A-AS1 facilitates hepatocellular carcinoma progression by binding with NOP58 to enhance the mRNA stability of FAM83A. Biosci Rep. https://doi. org/10.1042/bsr20192550

Heagerty PJ, Lumley T et al (2000) Time-dependent ROC curves for censored survival data and a diagnostic marker. Biometrics 56(2):337-344. https://doi.org/10.1111/j.0006-341x.2000.00337.x

Herbst RS, Morgensztern D et al (2018) The biology and management of non-small cell lung cancer. Nature 553(7689):446-454. https:// doi.org/10.1038/nature25183

Hua Q, Mi B et al (2020) Hypoxia-induced lncRNA-AC020978 promotes proliferation and glycolytic metabolism of non-small cell lung cancer by regulating PKM $2 / \mathrm{HIF}-1 \alpha$ axis. Theranostics 10(11):4762-4778. https://doi.org/10.7150/thno.43839

Huang GM, Zang HL et al (2020) LncRNA FAM83A-AS1 aggravates the malignant development of esophageal cancer by binding to miR-495-3p. Eur Rev Med Pharmacol Sci 24(18):9408-9415. https://doi.org/10.26355/eurrev_202009_23024

Jandura A, Krause HM (2017) The new RNA world: growing evidence for long noncoding RNA functionality. TIG 33(10):665-676. https://doi.org/10.1016/j.tig.2017.08.002

Ji X, Lin L et al (2020) Epigenetic-smoking interaction reveals histologically heterogeneous effects of TRIM27 DNA methylation on overall survival among early-stage NSCLC patients. Mol Oncol 14(11):2759-2774. https://doi.org/10.1002/1878-0261.12785

Jia J, Li H et al (2021) LncRNA FAM83A-AS1 promotes ESCC progression by regulating miR-214/CDC25B axis. J Cancer 12(4):1200-1211. https://doi.org/10.7150/jca.54007

Jiang R, Tang W et al (2009) A random forest approach to the detection of epistatic interactions in case-control studies. BMC Bioinform 10(Suppl 1):S65. https://doi.org/10.1186/1471-2105-10-s1-s65

Klipper-Aurbach Y, Wasserman M et al (1995) Mathematical formulae for the prediction of the residual beta cell function during the first two years of disease in children and adolescents with insulin-dependent diabetes mellitus. Med Hypothes 45(5):486-490. https://doi.org/10.1016/0306-9877(95)90228-7

Li J, Li X et al (2019) Gene-environment interaction in the era of precision medicine. Cell 177(1):38-44. https://doi.org/10.1016/j. cell.2019.03.004

Liang C, Qi Z et al (2018) Long non-coding RNA PCAT-1 in human cancers: a meta-analysis. Clin Chim Acta 480:47-55. https://doi. org/10.1016/j.cca.2018.01.043

Mok TS, Wu YL et al (2009) Gefitinib or carboplatin-paclitaxel in pulmonary adenocarcinoma. N Engl J Med 361(10):947-957. https:// doi.org/10.1056/NEJMoa0810699

Mok TSK, Wu YL et al (2019) Pembrolizumab versus chemotherapy for previously untreated, PD-L1-expressing, locally advanced or metastatic non-small-cell lung cancer (KEYNOTE-042): a randomised, open-label, controlled, phase 3 trial. Lancet (london,
England) 393(10183):1819-1830. https://doi.org/10.1016/S01406736(18)32409-7

Naidu S, Shi L et al (2017) PDGFR-modulated miR-23b cluster and miR-125a-5p suppress lung tumorigenesis by targeting multiple components of KRAS and NF-kB pathways. Sci Rep 7(1):15441. https://doi.org/10.1038/s41598-017-14843-6

Ren Y, Zhao C et al (2021) RPARP-AS1/miR125a-5p axis promotes cell proliferation, migration and invasion in colon cancer. Onco Targ Ther 14:5035-5043. https://doi.org/10.2147/ott.S304494

Robinson MD, Oshlack A (2010) A scaling normalization method for differential expression analysis of RNA-seq data. Genome Biol 11(3):R25. https://doi.org/10.1186/gb-2010-11-3-r25

Schmitt AM, Chang HY (2016) Long noncoding RNAs in cancer pathways. Cancer Cell 29(4):452-463. https://doi.org/10.1016/j.ccell. 2016.03.010

Shi R, Jiao Z et al (2019) Long noncoding antisense RNA FAM83AAS 1 promotes lung cancer cell progression by increasing FAM83A. J Cell Biochem 120(6):10505-10512. https://doi.org/ $10.1002 /$ jcb. 28336

Sung H, Ferlay J et al (2021) Global cancer statistics 2020: GLOBOCAN estimates of incidence and mortality worldwide for 36 cancers in 185 countries. CA Cancer J Clin 71(3):209-249. https:// doi.org/10.3322/caac. 21660

Travis WD (2020) Lung cancer pathology: current concepts. Clin Chest Med 41(1):67-85. https://doi.org/10.1016/j.ccm.2019.11.001

Trerotola M, Relli V et al (2015) Epigenetic inheritance and the missing heritability. Hum Genom 9(1):17. https://doi.org/10.1186/ s40246-015-0041-3

Wang L, Cho KB et al (2019) Long noncoding RNA (lncRNA)-mediated competing endogenous RNA networks provide novel potential biomarkers and therapeutic targets for colorectal cancer. Int $\mathbf{J}$ Mol Sci. https://doi.org/10.3390/ijms20225758

Wei Y, Zhang X (2016) Transcriptome analysis of distinct long noncoding RNA transcriptional fingerprints in lung adenocarcinoma and squamous cell carcinoma. Tumour Biol. https://doi.org/10. 1007/s13277-016-5422-2

Wu YL, Zhou C et al (2014) Afatinib versus cisplatin plus gemcitabine for first-line treatment of Asian patients with advanced non-smallcell lung cancer harbouring EGFR mutations (LUX-Lung 6): an open-label, randomised phase 3 trial. Lancet Oncol 15(2):213222. https://doi.org/10.1016/S1470-2045(13)70604-1

Wu Y, Wang PS et al (2018) Genomewide identification of a novel sixLncRNA signature to improve prognosis prediction in resectable hepatocellular carcinoma. Cancer Med 7(12):6219-6233. https:// doi.org/10.1002/cam4.1854

Wu L, Wen Z et al (2021) A novel autophagy-related lncRNA survival model for lung adenocarcinoma. J Cell Mol Med 25(12):56815690. https://doi.org/10.1111/jcmm.16582

$\mathrm{Xu}$ YH, Deng JL et al (2019) Long non-coding RNAs in prostate cancer: functional roles and clinical implications. Cancer Lett 464:37-55. https://doi.org/10.1016/j.canlet.2019.08.010

Zhang R, Lai L et al (2019a) SIPA1L3 methylation modifies the benefit of smoking cessation on lung adenocarcinoma survival: an epigenomic-smoking interaction analysis. Mol Oncol 13(5):12351248. https://doi.org/10.1002/1878-0261.12482

Zhang R, Lai L et al (2019b) EGLN2 DNA methylation and expression interact with HIF1A to affect survival of early-stage NSCLC. Epigenetics 14(2):118-129. https://doi.org/10.1080/15592294.2019. 1573066

Zhang G, Wang Q et al (2019c) LncRNA FENDRR suppresses the progression of NSCLC via regulating miR-761/TIMP2 axis. Biomed Pharmacother 118:109309. https://doi.org/10.1016/j.biopha.2019.109309

Zhang R, Chen C et al (2020a) Independent validation of early-stage NSCLC prognostic scores incorporating epigenetic and transcriptional biomarkers with gene-gene interactions and main effects. Chest. https://doi.org/10.1016/j.chest.2020.01.048 
Zhang X, Xie K et al (2020b) Role of non-coding RNAs and RNA modifiers in cancer therapy resistance. Mol Cancer 19(1):47. https://doi.org/10.1186/s12943-020-01171-z

Zhu L, Guo Q et al (2018) CTD-2020K17.1, a novel long non-coding rna, promotes migration, invasion, and proliferation of serous ovarian cancer cells in vitro. Med Sci Monit Int Med J Exp Clin Res 24:1329-1339. https://doi.org/10.12659/msm.908456
Publisher's Note Springer Nature remains neutral with regard to jurisdictional claims in published maps and institutional affiliations. 